\title{
Prevention and management of infection associated with transrectal ultrasound guided prostate biopsy in Ireland
}

\author{
Clíodhna Frances Ní Bhuachalla, Eileen Nolan, David Galvin, Claire M Buckley, Fidelma Fitzpatrick \\ National Cancer Control Programme (NCCP) National Prostate Biopsy Infection Project Board, \\ NCCP, Dublin, Ireland
}

doi: 10.3396/IJIC.v11i4.031.15

\begin{abstract}
The incidence of transrectal ultrasound (TRUS)-guided prostate biopsy associated infection is reportedly increasing, particularly antimicrobial resistant (AMR) infections. To inform development of an inaugural national policy on prevention and management of infection post TRUS biopsy, we conducted a national survey of ten prostate cancer centres that perform approximately $90 \%$ of public prostate biopsies in Ireland. An on-line questionnaire regarding prostate biopsy pathways, pre-biopsy AMR risk assessment and antimicrobial prophylaxis regimens, and post-biopsy infection surveillance and management was circulated to all centres. AMR organisms considered included Enterobacteriaceae (with particular reference to Escherichia coli) with fluoroquinolone and/or aminoglycoside resistance, extended spectrum betalactamase (ESBL) or carbapenamase production. Data from $1^{\text {st }}$ January 2011 to $30^{\text {th }}$ June 2013 were collected retrospectively from July to September 2013. Data were analysed using Microsoft Excel ${ }^{\circledR}$ software. The results of this survey demonstrated marked variation of practices nationally. Three centres reported risk assessing for AMR colonisation pre-biopsy. AMR screening was not conducted routinely in any centre. Antimicrobial prophylaxis regimens, surveillance programmes and empiric therapy guidelines for sepsis also varied between centres. A range of infectious complications were reported, both bloodstream infection (BSI) and non-BSI, however, due to use of non-standardised case definitions, national infection rates could not be generated. At the time of the survey, there were no Irish guidelines and centres followed American and/or European
\end{abstract}

\section{Corresponding Author}

Dr C.F. Ni Bhuachalla

NCCP, King's Inns House, 200 Parnell Street, Dublin1, Ireland.

Email: c_ni_bhuachalla@hotmail.com 
guidelines. Following our survey the National Cancer Control Programme published a "National Policy on the Prevention and Management of Infection post Transrectal Ultrasound-guided Prostate Biopsy" in June 2014 to assist in standardising practice and reducing risk of infectious complications.

Key words: Transrectal ultrasound-guided (TRUS) prostate biopsy; Prostate; Infections; Drug resistance, microbial, Antibiotic prophylaxis; Epidemiological surveillance; disease management

\section{Introduction}

Transrectal ultrasound (TRUS)-guided prostate biopsy is a standard diagnostic tool for prostate cancer. ${ }^{1}$ Public Rapid Access Prostate Clinics (RAPC), established by the Irish National Cancer Control Program (NCCP) in 2009, facilitate same day management of suspected prostate cancer and target men with indications for prostate biopsy. ${ }^{2}$ This one-stop diagnostic model facilitates rapid assessment and diagnosis, reducing patient anxiety and streamlining prostate cancer diagnosis and management.

Infectious complications following TRUS biopsy are increasing, specifically antimicrobial resistant (AMR) infections, and include urinary tract infection (UTI), prostatitis, bloodstream infection (BSI), and severe sepsis. ${ }^{3}$ Escherichia coli causes approximately $75 \%-90 \%$ of infections with increasing reports of fluoroquinolone resistance and/or production of an extended spectrum ß-lactamase (ESBL). ${ }^{3}$ AMR infection risk factors include recent fluoroquinolones, AMR colonisation or infection and recent international travel. ${ }^{1,3-5}$ Recent Irish AMR data demonstrated approximately $26.2 \%$ of invasive $E$. coli isolates tested were resistant to ciprofloxacin, approximately $10.1 \%$ of invasive $E$. coli isolates tested were found to be ESBLproducing and the first carbapenamase-producing $E$. coli from an invasive infection was confirmed. ${ }^{6}$

In light of reported increased incidence of infectious complications, and absence of Irish TRUS biopsy data, we conducted a national survey of public prostate cancer centres. The aim of this survey was to ascertain national practices to inform development of a national policy on prevention and management of infection post-TRUS biopsy.

\section{Methods}

An on-line questionnaire regarding prostate biopsy pathways, pre-biopsy AMR risk assessment and antimicrobial prophylaxis, and post-biopsy infection surveillance and management was circulated to ten public prostate cancer centres (all 8 NCCP centres and the 2 largest centres of 15 other public hospitals performing TRUS biopsies). These ten centres perform approximately $90 \%$ of public prostate biopsies nationally. Data from 1 $1^{\text {st }}$ January 2011 to $30^{\text {th }}$ June 2013 were collected retrospectively from July to September 2013 using SurveyMonkey ${ }^{\circledR}$. Data were analysed using Microsoft Excel ${ }^{\circledR}$ software.

Gathering and analysing national cancer data is consistent with the standard works and terms of reference of the NCCP thus institutional review board approval was deemed not to be required.

\section{Results}

All ten centres responded. All centres performed TRUS biopsies on-site, four also performed transperineal prostate biopsies. Biopsies were performed in interventional radiology $(n=5)$, RAPC facilities $(n=3)$ or urology outpatients $(n=2)$. All centres used oral fluoroquinolone antimicrobial prophylaxis; ciprofloxacin $(n=8)$, ofloxacin $(n=2)$. Five centres used a second agent; intravenous (IV) gentamicin, IV amikacin or oral metronidazole. Antimicrobial prophylaxis dosing schedules varied; single dose $(n=2), 24$ hours $(n=4), 48$ hours $(n=1), 72$ hours $(n=2), 5$ days $(n=1)$. Dosing of ciprofloxacin varied with either 500mg $(n=4)$ or $750 m g(n=4)$ prescribed.

No centre routinely performed pre-procedure AMR screening though one was conducting a pilot study on ESBL Enterobacteriaceae rectal screening. Three centres reported using a formal risk assessment tool to assess for AMR colonisation pre-biopsy. Risk factors assessed are described in Table I. No formal risk assessment tools or written protocols were returned.

Seven centres reported having a post-biopsy infection surveillance programme in place, although none 


\section{Table I. Risk assessment for AMR colonisation pre-biopsy}

\begin{tabular}{ll} 
Number of centres & Risk factor assessed \\
\hline $\mathrm{n}=3$ & - Previous AMR colonisation \\
& - Immunocompromise \\
\hline $\mathrm{n}=2$ & - Recent fluoroquinolone use \\
& - Previous antimicrobials \\
& - Previous urological procedure \\
\hline $\mathrm{n}=1$ & - Previous post-biopsy sepsis \\
& - Presence of indwelling material \\
& - Renal tract abnormality \\
& - Recent hospitalisation \\
& - Age \\
\hline
\end{tabular}

used standardised internationally comparable case definitions for infection categorisation. Methodology for infection follow-up varied and included telephone follow-up $(n=3)$, clinic review $(n=5)$ or a combination of methods $(n=2)$. Table II summarises infectious complications reported.

Seven centres had a protocol for infection management post-TRUS biopsy. Empiric antimicrobial prescribing guidelines varied; meropenem $(n=3)$, meropenem with gentamicin $(n=1)$, co-amoxiclav with gentamicin $(n=1)$ or piperacillin/tazobactam $(n=1)$. Empiric use of ciprofloxacin or amikacin was not reported. Four centres recommended prior discussion of empiric antimicrobial therapy with a clinical microbiologist or infectious diseases physician.

\section{Discussion}

This national survey revealed marked variation in practices in Irish centres performing TRUS biopsies. While all centres used fluoroquinolone prophylaxis in line with international guidelines ${ }^{4,5}$ there was no standardisation in relation to pre-biopsy AMR assessment and prophylaxis regimens, and post-biopsy surveillance and infection management.

\section{Table II. Number of prostate biopsies performed and post-biopsy infectious complications reported in ten Irish prostate cancer centres from 1st January 2011- 30th June 2013}

\begin{tabular}{|c|c|c|c|}
\hline Year & 2011 & 2012 & 2013* \\
\hline Number of prostate biopsies performed & $\begin{array}{r}3,466 \\
(\mathrm{n}=8)\end{array}$ & $\begin{array}{r}3,771 \\
(n=9)\end{array}$ & $\begin{array}{r}2,338 \\
(\mathrm{n}=7)\end{array}$ \\
\hline \multicolumn{4}{|l|}{ Number of post-biopsy BSI ${ }^{1}$} \\
\hline $0-5 \mathrm{BSI}$ & 3 centres & 3 centres & 5 centres \\
\hline 6-10 BSI & 1 centre & 3 centres & 0 \\
\hline$\geq 10 \mathrm{BSI}$ & 1 centre & 1 centre & 1 centre \\
\hline \multicolumn{4}{|l|}{ Number of post-biopsy non BSI² } \\
\hline 0-5 non BSI & 2 centres & 1 centre & 4 centres \\
\hline 6-10 non BSI & 1 centre & 3 centres & 0 \\
\hline$>10$ non BSI & 0 & 2 centres & 0 \\
\hline
\end{tabular}


TRUS biopsy is a widely performed, generally safe elective procedure but can be associated with infectious complications. Post-TRUS biopsy UTI rates of $2-6 \%$ are reported, with $30-50 \%$ of those patients developing BSI. A quarter of patients hospitalised with post-TRUS biopsy $E$. coli BSI had severe sepsis requiring intensive care unit admission. ${ }^{3}$ True incidence of infection may be underestimated, as reports usually concentrate on hospitalised patients rather than primary care patients (e.g., while $4.2 \%$ of patients had a fever in the two weeks post-procedure, only $0.8 \%$ were hospitalised). ${ }^{3}$

Irish prostate centres reported a small but significant number of post-TRUS biopsy infections. As standardised case definitions for infection surveillance were not employed, we could not produce Irish infection rates for benchmarking with other countries. However, national AMR surveillance data indicates that AMR Enterobacteriaceae spp. BSI is increasing. ${ }^{6}$ Standardising national surveillance methodology permits meaningful comparison of infection rates and analysis of outcomes. We recommend that Irish centres systematically capture UTI and BSI post-TRUS biopsy, using standardised European case definitions from 'Hospitals in Europe Link for Infection Control Surveillance (HELICS)' standards to enable comparison with other European centres, ${ }^{7}$ and conduct systems analysis of all BSI. The NCCP national policy outlines a national surveillance framework. ${ }^{8}$

Irish centres did not routinely screen patients for AMR and three centres (30\%) risk assessed patients. While individual studies indicate the usefulness of pre-biopsy rectal AMR screening to guide targeted antimicrobial prophylaxis, ${ }^{9}$ currently this approach is not recommended in international guidelines. ${ }^{4}$ Others recommend that urologists consider AMR screening after risk assessment. ${ }^{5}$ Pre-biopsy AMR screening to direct antimicrobial prophylaxis could certainly be justified in light of increasing reports of post-prostate biopsy AMR infections. ${ }^{1,3}$ In order to evaluate the cost effectiveness of a national prostate biopsy AMR screening programme in Ireland, we would require standardised surveillance on the burden of infection and reconfiguration of existing care pathways of RAPCs to enable prior AMR screening. The optimal specimen type and laboratory protocol for screening has yet to be defined. ${ }^{3}$
Study results demonstrated varied prophylaxis regimens. There are no definitive data to support the use of three day over 1 day dosing regimens, or multiple over single dose schedules. ${ }^{10}$ Prophylaxis should commence within 60 minutes of the biopsy and be discontinued within 24 hours. ${ }^{4}$ For patients without risk factors for AMR Enterobacteriaceae we recommend single dose $750 \mathrm{mg}$ oral ciprofloxacin, 4,5 and a 2 drug regimen if AMR risk factors present. $^{8}$ Transperineal prostate biopsy is less frequently associated with infection, ${ }^{3}$ and may be the preferred approach where risk of sepsis is high.

Limitations of this study are inclusion of public data only and that national infection rates could not be generated from the survey data.

Although international guidelines recommend a standardised approach to TRUS biopsy we found considerable practice variation in Ireland. At the time of the survey, there were no Irish guidelines and centres followed American, ${ }^{4}$ and/or European guidelines. ${ }^{5}$ Following our survey the NCCP published a national policy on the prevention and management of infection post-TRUS biopsy in June $2014^{8}$ with several infectionrelated key performance indicators for quarterly national monitoring. Effective implementation of this policy requires clear communication between all stakeholders and should assist in reducing infectious complications by standardising practice.

\section{Acknowledgements}

- Members of the NCCP Prostate Cancer Infection Board.

- The ten prostate cancer centres that completed the national survey.

- All authors report no relevant conflicts of interest or financial support to disclose. 


\section{References}

1. Wagenlehner FM, Pilatz A, Waliszewski P, Weidner W, Johansen TE. Reducing infection rates after prostate biopsy. Nat Rev Urol 2014; 11(2): 80-86.

2. Department of Health National Cancer Strategy, 2006. http:// www.cancerscreening.ie/publications/CancerControlStrategy2006.pdf [Accessed January 31, 2015].

3. Williamson DA, Barrett LK, Rogers BA, Freeman JT, Hadway $\mathrm{P}_{L}$ Paterson DL. Infectious complications following transrectal ultrasound-guided prostate biopsy: new challenges in the era of multidrug resistant Escherichia coli. Clin Infect Dis 2013; 57(2): 267-274.

4. American Urological Association Education and Research, Inc. BestPracticePolicyStatementonUrologicSurgeryAntimicrobial Prophylaxis, 2014. https:/www.auanet.org/common/pdf/ education/clinical-guidance/Antimicrobial-Prophylaxis.pdf [Accessed January 31, 2015]

5. Grabe M, Bartoletti R, Bjerklund-Johansen TE, et al. European Association of Urology Guidelines on Urological Infections, 2013. http://www.uroweb.org/gls/pdf/19\%20Urological\%20 infections_LR.pdf [Accessed January 31, 2015]

6. Health Protection Surveillance Centre, European Antimicrobial Resistance Surveillance System Surveillance Reports, 2014. http://www.hpsc.ie/A-Z/MicrobiologyAntimicrobialResistance/European AntimicrobialResistanceSurveillanceSystemEARSS/EARSSSurveillanceReports/ [Accessed January 31, 2015]
7. European Centre for Disease Control and Prevention Hospitals in Europe Link for Infection Control through Surveillance (HELICS) HAIICU protocol, 2010. http:// www.ecdc.europa.eu/en/aboutus/calls/Procurement $\% 20$ Related\%20Documents/5_ECDC_HAIICU_protocol_v1_1.pdf [Accessed January 31, 2015]

8. National Cancer Control Programme (NCCP) National Prostate Biopsy Infection Project Board. National Policy on the Prevention and Management of Infection post Transrectal Ultrasound (TRUS) guided Prostate Biopsy, 2014. http://www. hse.ie/eng/services/list/5/nccp/pubs/guidelines/NCCP\%20 Management $\% 20$ of $\% 2$ infection $\% 20$ post $\% 20$ TRUS $\% 20$ biopsy\%20policy\%20document.pdf [Accessed January 31, 2015]

9. Taylor AK, Zembower TR, Nadler RB, et al. Targeted antimicrobial prophylaxis using rectal swab cultures in men undergoing transrectal ultrasound guided prostate biopsy is associated with reduced incidence of postoperative infectious complications and cost of care. / Urol 2012; 187(4): 12751279.

10. Zani EL, Clark OA, Rodrigues NN Jr. Antimicrobial prophylaxis for transrectal prostate biopsy. Cochrane Database Syst Rev 2011; May 11; (5): CD006576. 